\title{
Lymphoid interstitial pneumonia: clinical features, associations and prognosis
}

\author{
S-I. Cha*, M.B. Fessler", C.D. Cool ${ }^{\mp}$, M.I. Schwarz" and K.K. Brown"
}

ABSTRACT: Lymphoid interstitial pneumonia (LIP) is rare and its clinical course incompletely described. The aim of this study was to examine the clinical features, associations and prognosis of surgical lung biopsy-proven LIP. The study group consisted of 15 subjects encountered over a 14-yr period.

The majority of subjects were females $(n=11)$ and the mean age was 47 yrs (range 17-78 yrs). Underlying systemic immune disorders were frequent, including Sjögren's syndrome $(n=8)$, rheumatoid arthritis, systemic lupus erythematosus, polymyositis, common variable immunodeficiency and dysproteinaemia. Only three patients were classified as "idiopathic". Presenting symptoms were dominated by dyspnoea and cough. Restrictive physiology, reduced diffusion capacity $(62.5 \pm 18.4 \%$ predicted) and bronchoalveolar lavage lymphocytosis $(30.5 \pm 29.1 \%$ pred $)$ were noted. Thirteen patients received corticosteroid therapy. Of the nine whose response could be assessed, four showed clinical improvement and four were stable. Overall, median survival was $\mathbf{1 1 . 5}$ yrs. Of the seven patients who died, respiratory problems were the primary cause of death in three. Conversion to lymphoma was not identified.

In conclusion, histopathological lymphoid interstitial pneumonia is commonly associated with immune system dysregulation, with idiopathic lymphoid interstitial pneumonia being extremely rare. Clinical stability or improvement with corticosteroids can be expected; however, survival remains impaired.

KEYWORDS: Corticosteroid, lymphoid interstitial pneumonia, survival

ymphoid interstitial pneumonia (LIP) was originally described by LIEBOW and CARRINGTON [1] as a benign lymphoproliferative disorder limited to the lungs and characterised by diffuse infiltration of the alveolar septa by dense collections of lymphocytes admixed with plasma cells and other cellular elements (fig. 1a). In a subsequent investigation it has been described as a hyperplastic disorder of the mucosa-associated lymphoid tissues (MALT), lying on a spectrum of pathological abnormalities that extends from follicular bronchiolitis to pseudolymphoma to LIP [2]. In some classification schemes it has been considered a preneoplastic condition with a high likelihood of progression to lymphoma [3]; however, immunohistochemical and molecular analyses indicate that malignant transformation is unusual $[4,5]$.

Histopathological LIP is a form of interstitial pneumonia that is uncommonly seen and its described clinical course is highly variable [6]. Radiographic features are somewhat nonspecific, though cysts associated with ground-glass infiltrates in a nonsmoker are a suggestive pattern (fig. 1b) [7]. The pathological finding has been associated with a variety of clinical conditions, such as connective tissue disorders, especially Sjögren's syndrome, and other immune system abnormalities, such as dysproteinaemia, AIDS and bone marrow transplantation [8]. Once underlying systemic diseases have been excluded, a diagnosis of "idiopathic" LIP can be made, and is classified as an idiopathic interstitial pneumonia (IIP) [9]. As earlier reports of the clinical features of LIP included subjects with low-grade lymphoma $[2,8,10,11]$, in the present study the authors explored the prospectively collected clinical database to review their experience of the clinical features and associations, response to treatment and prognosis of this histopathological entity.

\section{METHODS}

\section{Subject selection}

The present authors explored the prospectively collected clinical database of the Interstitial Lung Disease Program at the National Jewish Medical and Research Center (Denver, CO, USA) for patients seen between January 1985 and December 1999. Informed consent was obtained from each patient and the Institutional Human
AFFILIATIONS

${ }^{*}$ Dept of Internal Medicine, Kyungpook National University Hospital, Daegu, South Korea. \# Dept of Medicine, Division of Pulmonary Sciences and Critical Care Medicine, and

'Depts of Pathology and Medicine, University of Colorado Health Sciences University, National Jewish Medical and Research Center Denver, CO, USA.

CORRESPONDENCE

K.K. Brown

ILD Program

National Jewish Medical and

Research Center

1400 Jackson Street

Denver

CO 80206

USA

Fax: 13032702240

E-mail: brownk@njc.org

Received:

June 302005

Accepted after revision:

March 132006 

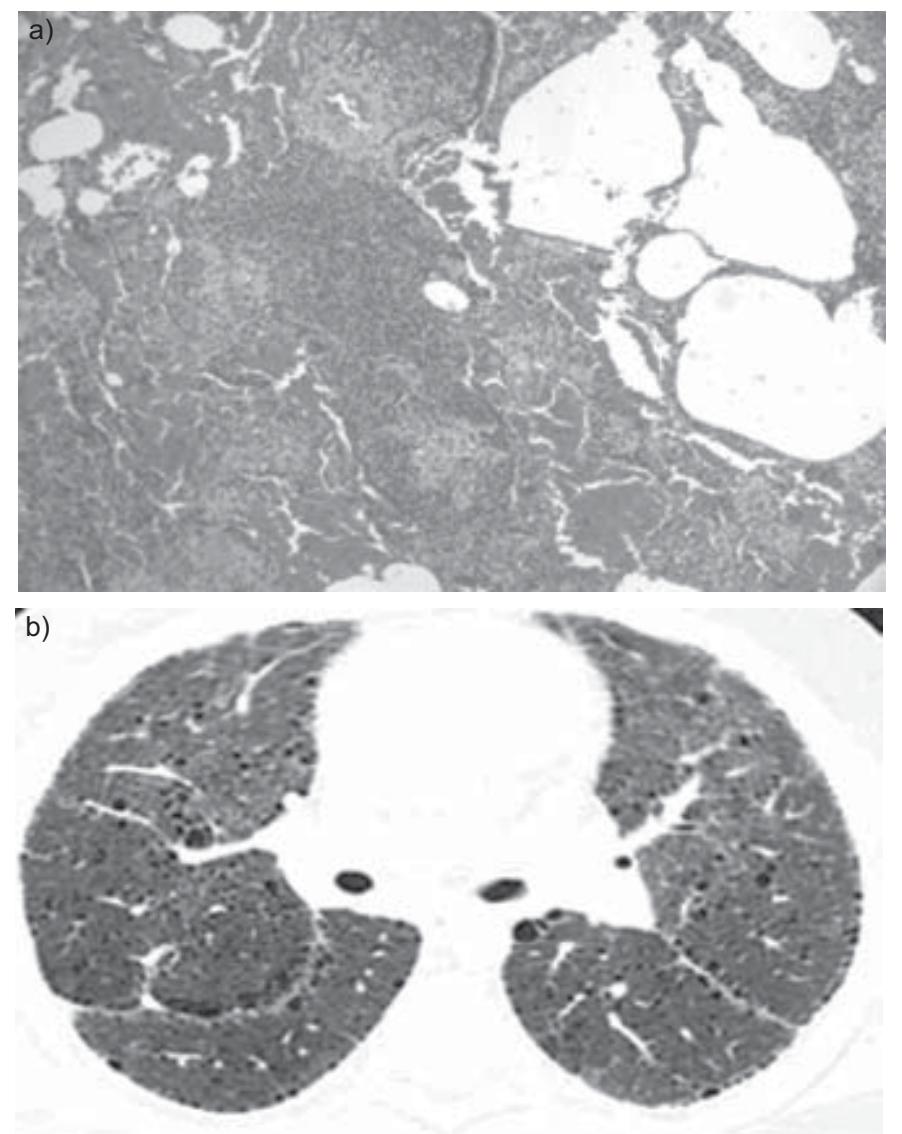

FIGURE 1. Pathological and computed tomographic findings of lymphoid interstitial pneumonia. a) Histopathological features include diffuse lymphocytic infiltrates with granuloma formation. b) Computed tomographic findings are nonspecific but include mixed alveolar interstitial infiltrates and thin-walled cysts.

Subject Review Committee (National Jewish Medical and Research Center) approved the protocol. Out of a total of 2,989 subjects (1,167 surgical lung biopsies) enrolled, 15 patients with histopathologically confirmed LIP were identified. An expert pulmonary pathologist (C.D. Cool) confirmed the pathological diagnosis by reviewing the original biopsy material in all subjects. Diagnosis was made via open lung biopsy $(n=11)$, video-assisted thoracoscopic surgery $(n=3)$ and transbronchial lung biopsy $(\mathrm{n}=1)$ with consistent high-resolution computed tomographic scanning [7]. All subjects were HIV negative. Subjects were classified as: current smokers, if they had smoked cigarettes regularly within the previous year; former smokers, if they had not smoked cigarettes in the previous year but had smoked in the past; and never-smokers.

\section{Clinical assessment}

A modified American Thoracic Society (ATS) questionnaire was used to collect demographic and clinical information [12]. The degree of dyspnoea was determined using a previously described dyspnoea scale [12]. Survival was assessed up to April 2003. Deaths were identified through family or referring physician contact or by searching the national death registry. Patients were diagnosed with Sjögren's syndrome, systemic lupus erythematosis, polymyositis and rheumatoid arthritis if they met American College of Rheumatology criteria for the diagnosis.

\section{Pulmonary physiology and bronchoalveolar lavage}

Resting pulmonary function test and cardiopulmonary exercise testing were performed as previously described [12-18]. Bronchoscopy with bronchoalveolar lavage (BAL) was performed after informed consent had been given, as previously described [19].

\section{Assessment of response to treatment}

Six months to $1 \mathrm{yr}$ after initial evaluation, response to treatment according to the international consensus for idiopathic pulmonary fibrosis (IPF) was assessed [20]. Based on clinical, radiological and physiological changes, the response was classified as improved, stable or worse [20].

\section{Statistical analysis}

Data are expressed as mean \pm SD. The Kaplan-Meier method was used to estimate survival. Survival time was calculated as the number of years from the patient's initial visit until death or time of censoring.

\section{RESULTS \\ Clinical characteristics}

The subjects' clinical characteristics are summarised in table 1. The male to female ratio was 1:2.75 and subject age ranged 17$78 \mathrm{yrs}$. Three-quarters of the subjects were never-smokers $(73.3 \%)$ and one-quarter were former smokers. The duration of symptoms prior to evaluation ranged from 2 months to 12 yrs. Respiratory symptoms were more common than systemic complaints and breathlessness was found in all patients. Chest examination often revealed bibasilar crackles, while digital clubbing was rare.

\section{Associated illnesses}

Sjögren's syndrome was present in eight subjects (53.3\%). Three patients had either rheumatoid arthritis, systemic lupus erythematosus or polymyositis (table 2). Two patients had a history of hypothyroidism. One patient (No. 14) was diagnosed with common variable immunodeficiency (CVID) $1 \mathrm{yr}$ prior to the diagnosis of the lung disease. In the seven patients who had serum protein electrophoresis and immunoelectrophoresis, dysproteinaemia was detected in five, hypergammaglobulinaemia in four and hypogammaglobulinemia in one. Consequently, three patients were classified as having idiopathic LIP and one of them (No. 4) had monoclonal hypergammaglobulinaemia.

\section{Pulmonary physiology}

Values were consistent with restrictive ventilatory disorders, including reduced forced vital capacity (FVC) and forced expiratory volume in one second (FEV1), elevated FEV1/FVC, and reduced total lung capacity and thoracic gas volume. The uncorrected diffusing capacity of the lung for carbon monoxide was decreased and gas exchange during maximal exercise testing was abnormal (table 3 ).

\section{Analysis of BAL fluid}

The total white blood cell count increased and the predominant cell type was the lymphocyte (table 4). The number of 


\begin{tabular}{|c|c|}
\hline TABLE 1 Clinical c & Clinical characteristics of subjects \\
\hline Characteristic & \\
\hline Subjects & 15 \\
\hline \multicolumn{2}{|l|}{ Sex } \\
\hline Males & 4 \\
\hline Females & 11 \\
\hline Age yrs & $47.1 \pm 18.9$ \\
\hline Caucasians & $13(86.7)$ \\
\hline \multicolumn{2}{|l|}{ Smoking status } \\
\hline Never-smoker & $11(73.3)$ \\
\hline Former smoker & $4(26.7)$ \\
\hline \multicolumn{2}{|l|}{ Constitutional symptoms } \\
\hline Fatigue & $13(86.7)$ \\
\hline Fever & $5(33.3)$ \\
\hline Unintentional weight loss & $5(33.3)$ \\
\hline Arthralgia & $6(40.0)$ \\
\hline \multicolumn{2}{|l|}{ Respiratory symptoms } \\
\hline Duration yrs & $2.8 \pm 3.2$ \\
\hline Cough & $8(53.3)$ \\
\hline Sputum & $5(33.3)$ \\
\hline Dyspnoea & $11(100)$ \\
\hline Grade & $3-10$ \\
\hline Duration yrs & $2.7 \pm 3.2$ \\
\hline \multicolumn{2}{|l|}{ Physical findings } \\
\hline Basilar crackles & $11(73.3)$ \\
\hline Wheezing & $1(7.7)$ \\
\hline Clubbing & $2(13.3)$ \\
\hline
\end{tabular}

Data are presented as $n$, mean \pm SD and $n(\%)$, unless otherwise stated.

T-cells (CD3 positive) and B-cells (CD21 positive) was within the normal range but T-suppressor cells (CD8 positive) increased compared with T-helper cells (CD4 positive).

\section{Response to treatment and prognosis}

Fourteen of the 15 subjects were treated specifically for lung disease. One patient (No. 12) did not receive therapy because their lung involvement was symptomatically and physiologically mild and stable for several years. All 14 subjects were treated with corticosteroids and/or cytotoxic drugs, including cyclophosphamide, azathioprine and cyclosporin A (table 5). Of these, two patients also received colchicine. Initial treatment was with corticosteroids in 12 patients and corticosteroids combined with methotrexate in one patient. For patient No. 2, a course of chloroquine preceded corticosteroid treatment. Response to therapy was evaluable in 10 subjects, with eight $(89 \%)$ showing either a favourable $(n=4)$ or stable $(n=4)$ course after steroid therapy. One patient (No. 1) failed with both corticosteroid and colchicine treatment and underwent single lung transplantation. Cyclophosphamide, azathioprine and methotrexate were either combined with or replaced corticosteroids and the response was variable, as shown in table 5. Interestingly, the patient with CVID-related LIP (No. 14) did not initially show improvement to corticosteroids and methotrexate but did respond to cyclosporin A.

The median survival of the subjects was 11.5 yrs (fig. 2). At the last follow-up, seven patients had died; two from end-stage lung disease, one from lung infection after lung transplantation, three from nonpulmonary causes and one from unidentifiable causes (table 6). The progression of LIP to end-stage lung disease was, therefore, found in three cases (patient Nos 1,2 and 7). Malignant transformation to pulmonary lymphoma was not detected.

\section{DISCUSSION}

In the present study, the authors sought to define the clinical features, associations and prognosis of biopsy-proven histopathological LIP. As it is not routine at the National Jewish Medical and Research Center to perform surgical lung biopsy on all connective tissue disease patients with radiographic infiltrates, the present cohort may not be inclusive of all LIP encounters at the centre over the study period. The finding of LIP was characterised by female predominance, a frequent association with systemic immune disorders, restrictive physiology, BAL lymphocytosis and impaired survival. Only three out of 15 cases were classified as idiopathic LIP. In most patients, a response of stability or clinical improvement was noted with corticosteroids, and one case of treatment-refractory LIP that appeared to respond to cyclosporin A was documented.

Consistent with previous studies [11, 21], the risk of transformation to malignant lymphoma appeared to be low. Whilst no cases of transformation to pulmonary lymphoma were identified, one patient with Sjögren's syndrome developed MALT lymphoma of the parotid gland and underwent successful surgical resection. A larger number of patients, followed for longer periods of time, would be needed to answer this question definitively. Two cases were associated with breast cancer, which was diagnosed before or after diagnosis of LIP. However, the relationship between LIP and breast cancer is unclear.

Most patients (eight out of nine) treated with corticosteroids were clinically stable or showed improvement. To date, corticosteroids have usually been the primary therapeutic regimen in LIP [22], although there have been no controlled clinical trials because of its low incidence. It is unclear whether treatment alters the natural course of LIP [23]. Other immunosuppressive agents, such as cyclophosphamide, azathioprine and chlorambucil, have been used anecdotally [23]. In the present study, cyclophosphamide, azathioprine and colchicine were used but the response was variable. A recent report [24] suggested a role for cyclosporin A in CVID-related LIP. Cyclosporin A has a complex action but the principal immunosuppressive effect is inhibition of CD4-positive Thelper type 2 lymphocyte activation and the related release of interleukin-4 and -5 , thus inhibiting clonal expansion of T-cells without directly affecting B-cells [25]. In the present cohort, patient No. 14 had CVID-related LIP and improved clinically with cyclosporin A after no improvement with steroids.

In the present study, IIP idiopathic LIP appeared to be very rare, with only three patients seen in a busy tertiary referral interstitial lung disease programme over a 15 -yr period. It should also be acknowledged that the identification of these three cases represents a maximal estimate. As the evaluation of autoimmunity or immune dysregulation was not prospectively defined in these patients, the possibility that an otherwise 


\section{TABLE 2 Underlying conditions}

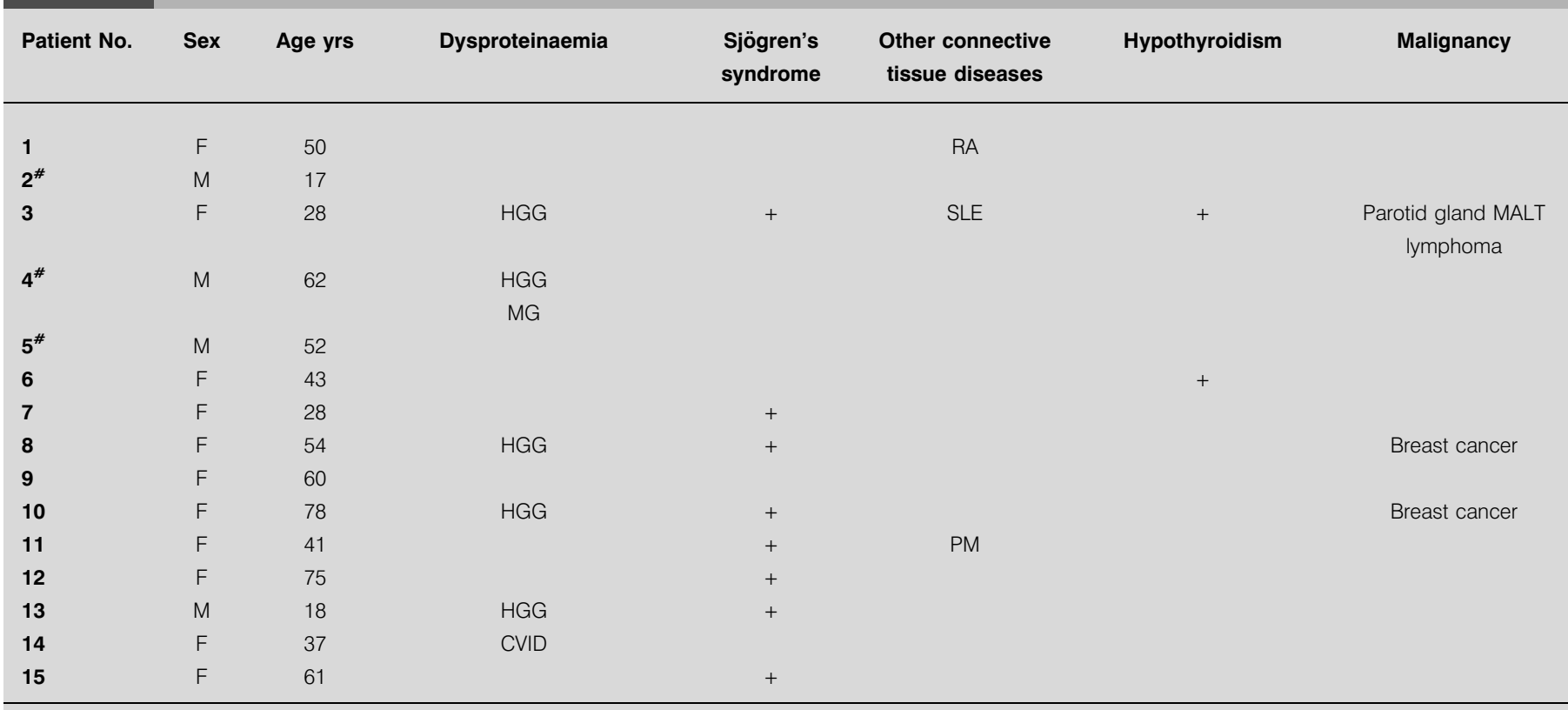

F: female; RA: rheumatoid arthritis; M: male; HGG: hypergammagobulinaemia; SLE: systemic lupus erythematosus; MALT: mucosal-associated lymphoid tissue; MG: monoclonal gammopathy; PM: polymyositis; CVID: common variable immunodeficiency. ${ }^{+}$: present. ${ }^{\#}$ : patients classified as having idiopathic lymphoid interstitial pneumonia.

occult systemic disorder was missed cannot be fully excluded. Of the three idiopathic cases, two (Nos 4 and 5) were alive at the final check, surviving $>10 \mathrm{yrs}$, with the response to steroids favourable in patient No. 4 and having failed in No. 5. The third patient (No. 2) had survived $>20$ yrs despite progression to end-stage lung disease. As patient No. 2

\section{TABLE 3 Lung function testing}

\begin{tabular}{lcc} 
Parameters & LIP & Subjects $\mathbf{~}$ \\
\hline FEV 1 \% pred & $67.3 \pm 18.4$ & 15 \\
FVC \% pred & $65.0 \pm 15.0$ & 15 \\
FEV 1 FVC \% & $80.3 \pm 5.0$ & 15 \\
TLC \% pred & $78.2 \pm 16.1$ & 13 \\
Vtg \% pred & $77.5 \pm 16.8$ & 13 \\
RV \% pred & $120.5 \pm 36.3$ & 13 \\
Raw \% pred & $135.1 \pm 56.8$ & 13 \\
DL,Co \% pred & $62.5 \pm 18.4$ & 13 \\
DL,Co/VA \% pred & $93.6 \pm 22.2$ & 13 \\
PA-a, O & & \\
$\quad$ Resting mmHg & $17.2 \pm 8.6$ & 12 \\
Peak exercise mmHg & $42.9 \pm 10.5$ & 10 \\
\hline
\end{tabular}

Data are presented as mean $\pm S D$, unless otherwise stated. LIP: Iymphoid interstitial pneumonia; FEV1: forced expiratory volume in one second; \% pred: per cent predicted; FVC: forced vital capacity; TLC: total lung capacity; Vtg: thoracic gas volume; RV: residual volume; Raw: airway resistance; $D L, C O$ : diffusing capacity of the lung for carbon monoxide; $V A$ : alveolar volume; $\mathrm{PA}_{\mathrm{A}-\mathrm{a}} \mathrm{O}_{2}$ : alveolar-arterial oxygen tension difference. $1 \mathrm{mmHg}=0.133 \mathrm{kPa}$. underwent open lung biopsy and received chloroquine and steroids $\sim 13$ yrs before referral to the present programme, the response to treatment was not evaluable. Whilst one out of three idiopathic LIP cases had died at the final follow-up, as opposed to six of the 12 nonidiopathic cases (fig. 2), the low numbers and retrospecttive nature of the present study precluded any firm statements on comparative prognosis. Similarly, it is intriguing that all three cases of idiopathic LIP were male, whereas 11 out of 12 nonidiopathic cases were female.

Despite the high prevalence of systemic immune disorders in the present cohort, three of the six deaths for which causes were identifiable were respiratory in nature. This finding

TABLE 4 Analysis of bronchoalveolar lavage fluid

\begin{tabular}{lcc} 
Parameter & Values & Subjects $\mathbf{n}$ \\
\hline WBC count $\times \mathbf{1 0}^{\mathbf{6}}$ & $60.6 \pm 40.5$ & 6 \\
Differential count \% & & \\
Alveolar macrophages & $44.5 \pm 28.6$ & 6 \\
Neutrophils & $17.5 \pm 17.3$ & 6 \\
Eosinophils & $7.5 \pm 7.0$ & 6 \\
Lymphocytes & $30.5 \pm 29.1$ & 6 \\
T-cells (CD3) & $75.7 \pm 27.7$ & 3 \\
T-helper (CD4) & $30.3 \pm 33.5$ & 3 \\
T-suppressor (CD8) & $37.3 \pm 33.7$ & 3 \\
B-cells (CD21) & $0.8 \pm 1.1$ & 6 \\
\hline
\end{tabular}

Data are presented as mean \pm SD. WBC: white blood cell. 
TABLE 5 Response to treatment in subjects in whom follow-up was available

\begin{tabular}{|c|c|c|c|c|c|}
\hline \multirow[t]{2}{*}{ Patient No. } & \multirow[t]{2}{*}{ Treatment $^{\#}$} & \multirow[t]{2}{*}{ Clinical response } & \multirow[t]{2}{*}{ Radiological response } & \multicolumn{2}{|c|}{ Physiological response } \\
\hline & & & & TLC and/or FVC & $D L, C O$ \\
\hline \multirow[t]{2}{*}{1} & Steroid & $\uparrow$ & $\rightarrow$ & $\rightarrow$ & $\rightarrow$ \\
\hline & Colchicine & & & & \\
\hline 3 & Steroid & $\uparrow$ & $\uparrow$ & $\uparrow$ & $\uparrow$ \\
\hline 6 & Steroid & NA & $\uparrow$ & $\uparrow$ & $\uparrow$ \\
\hline \multirow[t]{3}{*}{7} & Steroid & $\rightarrow$ & $\downarrow$ & $\rightarrow$ & $\rightarrow$ \\
\hline & Cyclophosphamide & $\downarrow$ & $\downarrow$ & $\rightarrow$ & $\rightarrow$ \\
\hline & Colchicine & $\uparrow$ & $\rightarrow$ & $\rightarrow$ & $\rightarrow$ \\
\hline 8 & Steroid & $\uparrow$ & $\rightarrow$ & $\downarrow$ & $\rightarrow$ \\
\hline 13 & Steroid & & & & \\
\hline \multirow[t]{2}{*}{15} & Steroid+MTX & $\uparrow$ & $\uparrow$ & $\downarrow$ & $\rightarrow$ \\
\hline & Cyclophosphamide & $\uparrow$ & $\uparrow$ & $\rightarrow$ & $\rightarrow$ \\
\hline
\end{tabular}

Response was defined according to the American Thoracic Society consensus criteria for monitoring idiopathic pulmonary fibrosis [20]. Clinical response was defined as a clinically significant change in dyspnoea and/or cough; radiographical response was defined response as a measureable change in radiological opacities; and physiological response was defined as either $\geqslant 10 \%$ change in forced vital capacity (FVC) and/or total lung capacity (TLC) or $\geqslant 15 \%$ change in diffusing capacity of the lung for carbon monoxide $(D L, C O)$. Response in all subjects was measured 6-12 months after diagnosis. TLC: total lung capacity; FVC: forced vital capacity; NA: not available; MTX: methotrexate. ${ }^{*}$ : consecutive therapies listed in chronological order; ${ }^{\bullet}$ : patients classified as having idiopathic lymphoid interstitial pneumonia. $\uparrow$ : improvement; $\rightarrow$ : no change; $\downarrow$ : decrease

suggests that LIP may have an independent impact on survival. Of interest in this regard is the fact that it has been reported that granulomatous-lymphocytic interstitial lung disease (GLILD), a classification that includes LIP, is associated with worsened survival among patients with an underlying diagnosis of CVID [26]. In the report by BATES et al. [26], CVID patients with GLILD were found to have a median survival of

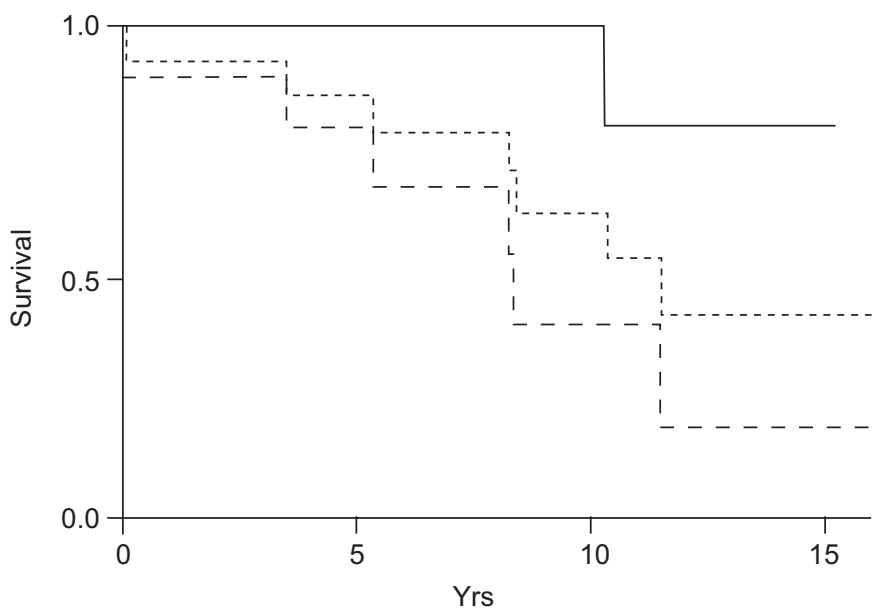

FIGURE 2. Survival curve of patients with lymphoid interstitial pneumonia (LIP). The median survival was $11.5 \mathrm{yrs}$. —_: idiopathic LIP; -----: LIP; - - -: nonidiopathic LIP.
$13.7 \mathrm{yrs}$, which is very similar to the median survival of 11.5 yrs noted for LIP in the present study.

The present study also sought to address some of the "areas of uncertainty" in LIP research proposed in the Joint Statement of

\section{TABLE 6 Prognosis and cause of death}

\begin{tabular}{lcc} 
Patient No. & Prognosis & Cause of death \\
\hline $\mathbf{1}$ & Deceased & Following lung transplant \\
$\mathbf{2}^{\#}$ & Deceased & End-stage lung disease \\
$\mathbf{3}$ & Alive & \\
$\mathbf{4}^{\#}$ & Alive & \\
$\mathbf{5}^{\#}$ & Alive & \\
$\mathbf{6}$ & Alive & \\
$\mathbf{7}$ & Deceased & End-stage lung disease \\
$\mathbf{8}$ & Deceased & Nonpulmonary cause \\
$\mathbf{9}$ & Alive & \\
$\mathbf{1 0}$ & Deceased & Congestive heart failure \\
$\mathbf{1 1}$ & Deceased & NA \\
$\mathbf{1 2}$ & Deceased & Nonpulmonary cause \\
$\mathbf{1 3}$ & Alive & \\
$\mathbf{1 4}$ & Alive & \\
$\mathbf{1 5}$ & Alive & \\
\hline
\end{tabular}

NA: not available. ${ }^{\#}$ : patients classified as having idiopathic lymphoid interstitial pneumonia. 
the ATS/European Respiratory Society [9], including the incidence and prevalence of LIP, the frequency of idiopathic LIP, its malignant potential and whether corticosteroids alter the natural history. The present data suggest that, given the rarity of this disease, a large multicentred, multinational effort will be necessary to answer the majority of these proposed questions.

In conclusion, histopathological lymphoid interstitial pneumonia is an uncommon interstitial lung disease, with idiopathic lymphoid interstitial pneumonia being extremely rare. In a similar manner to other interstitial lung diseases, physiological restriction and abnormal gas exchange are common and the expected bronchoalveolar lavage lymphocytosis is present. Clinical stability or improvement in response to corticosteroids may be expected, however survival is impaired. Large multicentre trials will be necessary to further understanding of this disorder.

\section{REFERENCES}

1 Liebow A, Carrington CB. The interstitial pneumonias. In: Simon M, Potchen EJ, LeMay M, eds. Frontiers of Pulmonary Radiology. 1st Edn. New York, Grune \& Stratton, 1969; pp. 102-141.

2 Koss M, Hochholzer L, Langloss J, et al. Lymphoid interstitial pneumonia: clinicopathological and immunopathologic findings in 18 cases. Pathology 1987; 19: 178-185.

3 Koss MN. Pulmonary lymphoid disorders. Semin Diagn Pathol 1995; 12: 158-171.

4 Katzenstein ALA, Myers JL. Idiopathic pulmonary fibrosis: clinical relevance of pathologic classification. Am J Respir Crit Care Med 1998; 157: 1301-1315.

5 Kradin RL, Young RH, Kradin LA, Mark EJ. Immunoblastic lymphoma arising in chronic lymphoid hyperplasia of the pulmonary interstitium. Cancer 1982; 50: 1339-1343.

6 Teruya-Feldstein J, Temeck BK, Sloas MM, et al. Pulmonary malignant lymphoma of mucosa-associated lymphoid tissue (MALT) arising in a pediatric HIV positive patient. Am J Surg Pathol 1995; 19: 357-363.

7 Johkoh T, Muller NL, Pickford HA, et al. Lymphocytic interstitial pneumonia: thin-section CT findings in 22 patients. Radiology 1999; 212: 567-572.

8 Liebow A, Carrington C. Diffuse pulmonary lymphoreticular infiltrations associated with dysproteinemia. Med Clin North Am 1973; 57: 809-843.

9 American Thoracic Society/European Respiratory Society International Multidisciplinary Consensus Classification of the Idiopathic Interstitial Pneumonias. Am J Respir Crit Care Med 2002; 165: 277-304.

10 Travis WD, Galvin JR. Non-neoplastic pulmonary lymphoid lesions. Thorax 2001; 56: 964-971.
11 Strimlan CV, Rosenow EC 3rd, Weiland LH, Brown LR. Lymphocytic interstitial pneumonitis. Ann Intern Med 1978; 88: 616-621.

12 Watters LC, King TE, Schwarz MI, Waldron JA, Stanford RE, Cherniack RM. A clinical, radiographic, and physiologic scoring system for the longitudinal assessment of patients with idiopathic pulmonary fibrosis. Am Rev Respir Dis 1986; 133: 97-103.

13 Morris JF, Koski A, Johnson LC. Spirometric standards for healthy non-smoking adults. Am Rev Respir Dis 1971; 613: 57-67.

14 Goldman HI, Becklake MR. Respiratory function tests. Normal values at medium altitude and the prediction of normal results. Am Rev Tuberc 1959; 79: 457-467.

15 Ogilvie CM, Forster RE, Blakemore WS, Morton JW. A standardized breathholding technique for the clinical measurement of the diffusing capacity of the lung for carbon monoxide. J Clin Invest 1957; 36: 1-17.

16 Crapo RO, Morris AH. Standardized single breath normal values for carbon monoxide diffusing capacity. Am Rev Respir Dis 1981; 123: 185-189.

17 Riley RL, Cournand A. Ideal alveolar air and the analysis of ventilation-perfusion relationships in the lungs. J Appl Physiol 1949; 1: 825-847.

18 Jones NL, Campbel EJM. Clinical Exercise Testing. Philadelphia, W.B. Saunders Co., 1982; p. 249.

19 Watters LC, King TE, Cherniack RM, et al. Bronchoalveolar lavage fluid neutrophils increase after corticosteroid therapy in smokers with idiopathic pulmonary fibrosis. Am Rev Respir Dis 1986; 133: 104-109.

20 American Thoracic Society. Idiopathic pulmonary fibrosis: diagnosis and treatment. International consensus statement. Am J Respir Crit Care Med 2000; 161: 646-664.

21 Fishback N, Koss M. Update on lymphoid interstitial pneumonitis. Curr Opin Pulm Med 1996; 2: 429-433.

22 Swigris JJ, Berry GJ, Raffin TA, Kuschner WG. Lymphoid interstitial pneumonia: a narrative review. Chest 2002; 122: 2150-2164.

23 Nicholson AG, Wotherspoon AC, Diss TC, et al. Reactive pulmonary lymphoid disorders. Histopathology 1995; 26: 405-412.

24 Davies CWH, Gray W, Gleeson FV, Chapel HM, Davies RJO. Lymphoid interstitial pneumonitis associated with common variable hypogammaglobulinemia treated with cyclosporin A. Thorax 2000; 55: 88-90.

25 Lynch JP, McCune WJ. Immunosuppressive and cytotoxic pharmacology for pulmonary disorders. Am J Respir Crit Care Med 1997; 155: 395-420.

26 Bates CA, Ellison MC, Lynch DA, Cool CD, Brown KK, Routes JM. Granulomatous-lymphocytic lung disease shortens survival in common variable immunodeficiency. J Allergy Clin Immunol 2004; 114: 415-421. 